\title{
Clinical manifestations of prune belly syndrome
}

\author{
Authors: R Chhabra, ${ }^{1}$ A Awan, ${ }^{2,3}$ C Stapleton, ${ }^{1}$ G Cavalleri ${ }^{1}$ and P Conlon ${ }^{1,2}$
}

\section{Aims}

We reviewed a cohort of 13 patients with prune belly syndrome (PBS) in our centres and analysed their clinical characteristics, diagnostics, treatment, complications and long-term clinical outcome including renal transplantation.

\section{Methods}

All clinical records of patients with PBS, treated or followed up in a tertiary renal transplant centre and two main paediatric centres between 1968 and 2015, were reviewed retrospectively. A total of 13 patients with PBS were identified and included in the study.

\section{Results}

In our centre, most patients were diagnosed at birth (84.6\%). Cryptorchidism was present in seven patients (53.8\%); lax abdominal wall was present in three patients $(23 \%)$. All patients had genitourinary abnormalities, obstruction being the predominant anomaly, present in $53.8 \%$.

All patients developed end-stage kidney disease (ESKD) at a mean age of 11.4 years (standard deviation 8.8 years). All patients with ESKD were transplanted. Mean age at first transplant was 12.9 years. One patient received a pre-emptive transplant at age 10 . Three patients $(23 \%)$ required a second transplant. Eleven patients $(84.6 \%)$ of 13 are alive. Two patients $(15.3 \%)$ are deceased due to unrelated causes. Ten of the 11 patients $(90.9 \%)$ who are alive have a functioning transplant.

Graft survival was $86.5 \%$ (95\% confidence interval 55.8-96.5\%) at 5 years post transplant, with a graft half-life of 12.9 years. Patient survival was $100 \%$ at 5 years, with patient half-life estimated at 19.9 years. No disease-related complications were reported at the time of transplantation.

\section{Conclusions}

PBS is a complex disorder associated with variable severity and clinical manifestations. We demonstrate that successful treatment is possible even in cases of complex anomalies. We have collected DNA on familial trios and are embarking on a whole exon sequence study to identify underlying molecular mechanisms.

Authors: ${ }^{1}$ Beaumont Hospital, Dublin, Ireland; ${ }^{2}$ Temple Street Children's University Hospital, Dublin, Ireland; ${ }^{3}$ Our Lady's Children's Hospital, Crumlin, Dublin, Ireland 